\title{
THE EFFECTS OF TRICALCIUM PHOSPHATE AND NOVAMIN REMINERALIZING AGENTS ON MICROHARDNESS OF BLEACHED ENAMEL WITH 35\% HYDROGEN PEROXIDE-AN IN VITRO STUDY
}

\author{
Sahar Abd El Halim*
}

\begin{abstract}
Objectives: The aim of the present study was to evaluated the effects of remineralizing agents (Tricalcium phosphate and Novamin) on microhardness of bleached enamel surface with $35 \%$ hydrogen peroxide (in office).

Materials and Methods: Forty human permanent maxillary premolars free from caries or enamel malformation were used. The teeth were sectioned transversally across the cemento-enamel junction, and specimens were embedded in self-cure acrylic. The labial enamel surface faced upward and then they were ground flat and polished with silicon carbide abrasive papers. A total specimen were prepared and kept in deionized water. Then the specimens were drying subjecting them for baseline microhardness testing (control). The dental bleaching treatment was performed just on the labial enamel surface of the specimens using a commercial $35 \%$ hydrogen peroxide Whitness HP bleaching gel (in-office ).It was used according to the manufacture instruction. Microhardness was tested for all specimens after bleaching. Specimens were divided into two groups according to remineralizing agent $n=20$, Group I: Treated by NUPRO Sensodyne with NovaMin, Group II: Treated by Clinpro ${ }^{\mathrm{TM}}$ White Varnish Tri-Calcium Phosphate. The remineralizing agent was applied for three minutes per day. Two remineralizing agents were applied for fourteen consecutive days. After each application procedure, the specimens were washed with deionized water and then kept in artificial saliva. At the end of the fourteen days the remineralized specimens were subjected to microhardness testing and values were recorded. All the data were analyzed by ANOVA and Tukey's post hoc test.
\end{abstract}

Results: Results revealed that a statistically significant decrease on mean microhardness values after enamel bleaching compared to control, followed by a significant increase on mean microhardness after the application of the both remineralizing agents. One way-ANOVA showed a statistically insignificant difference between NUPRO Sensodyne with NovaMin and Clinpro ${ }^{\mathrm{TM}}$ White Varnish at $\mathrm{p} \geq 0.05$.

Conclusions: Based on the results of this in vitro study both Tricalcium phosphate and Novamin are effective in remineralization of bleached enamel surface and no significant difference was detected in their efficacy.

* Associate Professor, Operative Dentistry Department, Misr University for Science and Technology, Egypt 


\section{INTRODUCTION}

Dental bleaching has been described as a conservative and simple procedure for aesthetic restoration. Recently, there are many bleaching agents commercially available with various components and concentrations. ${ }^{[1-2]}$.

Evidence shows that the enamel structure will be changed when exposed to different bleaching agents, which affecting its composition and morphology ${ }^{[3]}$. Various studies show that bleaching decreased the enamel mineral content, especially lowering calcium and phosphate, so altering the chemical, structural, and teeth's mechanical properties, due to demineralization. These changes include decreased microhardness and increased porosity and permeability ${ }^{[4-5]}$. Also, loss of enamel hardness was detected, as bleaching agents are not limited to the enamel surface alone, but can also be present in the sub surface within in the outermost layer ${ }^{[6-7]}$. Subsequently, bleaching affects the organic and the inorganic components of enamel, increasing the capability to erosion, decreasing fracture stability and/or decrease abrasion resistance of the bleached dental hard tissues ${ }^{[8]}$.

In order to avoid or reduce the micro-structural changes on enamel during and after tooth bleaching, various materials and protocols have been used, such as Functionalized tricalcium phosphateis output by milling TCP with sodium lauryl sulfate. fTCP stabilizes fluoride in solution and preserve high concentration of calcium, phosphate and fluoride[9]. Implying of the functionalized TCP constituent in $\mathrm{NaF}$ formulations has been shown to produce stronger, more acid-resistant mineral relative to only fluoride in laboratory and clinical evaluations [10-11].As well as NovaMin is a synthetic mineral compound consisted of calcium, sodium, phosphorus, and silica that can enhance remineralization. It adheres to the tooth surface and constantly deposits crystalline hydroxyl-carbonate apatite (HCA). Microscopic particles of NovaMin liberate millions of mineral ions upon exposure to humidity, resulting in forming a firm hydroxyapatite (HA) layer on the surface of the enamel and dentin. This ability not only repairs initial carious lesions, but also remove tooth hypersensitivity ${ }^{[12-13]}$.

The current study evaluated effects of remineralizing agents (tricalcium phosphate and novamin) on microhardness of bleached enamel surface with $35 \%$ hydrogen peroxide (in office).

\section{MATERIALS AND METHODS}

\section{Teeth selection.}

Forty human permanent maxillary premolars free from caries or enamel malformation, indicated for extraction were included in the study. Extracted teeth were cleaned with scalar and pumice to get rid of debris and stains from enamel surface, after which the teeth were stored in distilled water containing thymol crystals during the interval between extraction and their use in this in vitro study.

\section{Specimens' preparation}

The teeth were sectioned transversally across the crown/root border, along the cemento-enamel junction, remnants of pulp discarded and specimens were embedded in self-cure acrylic (Rapid Repair, Detrey Dentsply Ltd, Surrey, U.K.).

The labial enamel surface faced upward and then they were ground flat and polished sequentially with $600,800,1,500$, and 3,000 grit silicon carbide abrasive papers (Silicium Carbide, Matador, Wasserfest 991A, Soft flex, 3M ESPE Co., St. Paul, MN, USA). A total specimen were prepared and kept in deionized water to avoid dehydration. Then the specimens were drying with absorbent paper before subjecting them for baseline microhardness testing (control)

\section{Teeth bleaching experiment}

The dental bleaching treatment was performed just on the labial enamel surface of the specimens using a commercial $35 \%$ hydrogen peroxide 
bleaching gel (in-office) Whitness HP (FGM, Produtos Odontológicos Ltda, Joinvile, Santa Catarina, Brazil) according to the manufacture instruction. The bleaching gel was applied over the surface of the specimens in a $2 \mathrm{~mm}$ thick layer (approximately $0.1 \mathrm{~g}$ ). It remained on the surface for $15 \mathrm{~min}$, being gently stirred after $5 \mathrm{~min}$ using a plastic instrument to dislodge the bubbles formed. The gel was removed using a vacuum aspirator and a new layer was applied following the same steps described. The second layer of gel was removed and a third layer was applied, in the same way as the previous two. This means that the bleaching gel remained on the enamel surface for a total of $45 \mathrm{~min}$, simulating the protocol based on the manufacture instructions. After bleaching, they were stored in distilled water and the bleaching was repeated twice with one week interval. Microhardness was tested for all specimens after bleaching.

\section{Remineralizing agent}

Specimens were divided into two groups according to remineralizing agent $n=20$

Group I: Treated by NUPRO Sensodyne with NovaMin (Prophylaxis Paste with NovaMin; Dentsply International, USA), which was applied using a rubber cup to each tooth for one min, with wobbling movements in clockwise direction, and were then allowed to stand for one min before rinsing, after that specimens were washed with deionized water for $30 \mathrm{~s}$ and dried for $30 \mathrm{~s}$.

Group II: Treated by Clinpro ${ }^{\mathrm{TM}}$ White Varnish (3M ESPE, 2510 Conway Avenue St. Paul, MN55144 -1000 USA) Tri-Calcium Phosphate, which was applied using applicator brush. The remineralizing agent was applied for three minutes per day.

Two remineralizing agents were applied for fourteen consecutive days. After each application procedure, the specimens were washed with deionized water and then kept in artificial saliva ( (0.4g NaCl-0.4g KCl-0.795g CaCl2.2H2O-0.69g
Na2HPO4- 0.005g Na2S. 9H2O-1g Urea + 1 L Deionized water) $\mathrm{pH}$ (7.03) at room temperature ${ }^{[14]}$. At the end of the fourteen days the remineralized specimens were subjected to microhardness testing and values were recorded.

\section{Microhardness testing}

Vickers hardness measurements

The surface hardness of the specimens was determined using a Digital Display Vickers Microhardness Tester (Model HVS-50, Laizhou Huayin Testing Instrument Co., Ltd., China) with a Vickers diamond indenter and a 20X objective lens. A load of $200 \mathrm{~g}$ was applied to the surface of the specimens for 15 seconds. Three indentations were equally placed over a circle of $1 \mathrm{~mm}$ in diameter at the middle third of the specimens. The diagonal lengths of the indentations were measured by a built-in scaled micrometer, and the Vickers values were converted into micro-hardness values.

Micro-hardness was obtained using the following equation:

\section{$H V=1.854 \mathrm{P} / \mathrm{d} 2$}

where:

$\mathbf{H V}$ is Vickers hardness in $\mathrm{Kgf} / \mathrm{mm} 2$;

$\mathbf{P}$ is the load in Kgf;

$\mathbf{d}$ is the average diagonal lengths in $\mathrm{mm}$.

\section{Statistical analysis}

Statistical analysis was then performed using a commercially available software program (SPSS 18; SPSS, Chicago, IL, USA). As data was parametric, significance of the difference in microhardness was evaluated using one way analysis of variance (ANOVA test). Duncan's post-hoc test was used for pair-wise comparison between the means when ANOVA test is significant. Independent t-test used to evaluate the different effect of the remineralizing agents on mean microhardness (VHN). 


\section{RESULTS}

\section{Microhardness}

Results revealed that a statistically significant decrease on mean microhardness values had resulted after enamel bleaching compared to control (unbleached enamel), followed by a significant increase on mean microhardness after the application of the both remineralizing agents. One way-ANOVA showed a statistically insignificant difference between NUPRO Sensodyne with
NovaMin and Clinpro ${ }^{\mathrm{TM}}$ White Varnish at $\mathrm{p} \geq 0.05$.

The greatest mean value was recorded in Novamin remineralizing agent. However, there was no significant difference between mean values recorded with both remineralizing agents and baseline (control) values. Means values recorded with both and remineralizing agents and at baseline were significantly greater than mean values recording after bleaching $(\mathrm{p}<0.0001)$, (Table1, Fig.1)

TABLE (1) Microhardness values (ANOVA test)

\begin{tabular}{|c|c|c|c|c|c|c|c|c|c|}
\hline & \multirow{2}{*}{ Mean } & \multirow{2}{*}{ Std. Dev } & \multirow{2}{*}{ Std. Error } & \multicolumn{2}{|c|}{$\begin{array}{l}\text { 95\% Confidence Interval } \\
\text { for Mean }\end{array}$} & \multirow{2}{*}{ Min } & \multirow{2}{*}{ Max } & \multirow{2}{*}{$\mathrm{F}$} & \multirow{2}{*}{$P$} \\
\hline & & & & $\begin{array}{l}\text { Lower } \\
\text { Bound }\end{array}$ & $\begin{array}{l}\text { Upper } \\
\text { Bound }\end{array}$ & & & & \\
\hline Control & $296.30^{\mathrm{a}}$ & 2.94 & 0.66 & 294.92 & 297.68 & 293.00 & 302.00 & 33.97 & $<0.0001 *$ \\
\hline After bleaching & $279.35^{\mathrm{b}}$ & 11.53 & 2.58 & 273.95 & 284.75 & 253.00 & 288.00 & & \\
\hline Novamin & $295.55^{\mathrm{a}}$ & 2.65 & 0.59 & 294.31 & 296.79 & 290.00 & 299.00 & & \\
\hline Tricalcium phosphate & $295.25^{\mathrm{a}}$ & 3.04 & 0.68 & 293.83 & 296.67 & 288.00 & 299.00 & & \\
\hline
\end{tabular}

*significant at $p<0.05$

Tukey's post hoc test: means with different superscript letter are significantly different

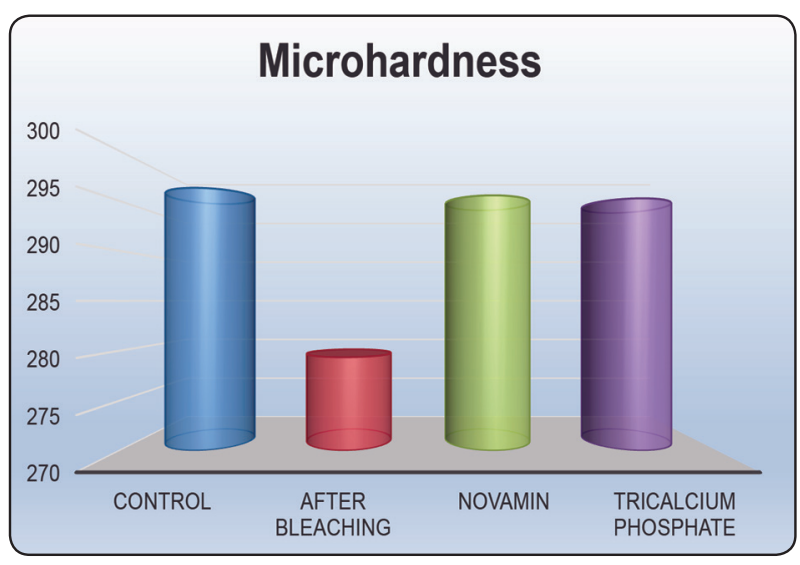

Fig. (1) Mean Microhardness values recorded at baseline (control), after bleaching and after remineralization

\section{DISCUSSION}

The current study examined the effect of remineralizing agents (Tricalcium Phosphate and Novamin) on microhardness of enamel surface after bleaching with $35 \%$ hydrogen peroxide (in office).

In contemporary years, bleaching gels containing high concentrations of hydrogen peroxide (HP, $35-45 \%$ ) have made in office bleaching techniques easier and more favorable. The advantage of this bleaching technique is its proper and immediate results with no need for further patient cooperation, in addition patient is comfort and minimum chair time ${ }^{[15]}$. 
Microhardness test is a simple and frequently used technique to determine the mechanical properties of enamel and dentine following the bleaching process ${ }^{[16]}$. In the present study VHN was selected over Knoop's because a square shape of indent resulted from VHN was easy and more precise to measure ${ }^{[17]}$.

As regards the microhardness, the results of the current study showed significant difference between control specimens (no treatment) and bleaching specimens. There was significant decrease in microhardness after bleaching.

These findings were in agreement with other studies that showed that Hydrogen peroxide induced enamel mineral loss after bleaching and this process can lead to demineralization of the enamel surface and compromise its mechanical properties. Hydrogen peroxide is one of the most used bleaching agents commercially available today and is a substance that is capable of significantly altering the enamel surface. Its action is generally linked to its oxidizing properties, which can break organic molecules claiming not to dissolve the enamel matrix, eventually producing clearer compounds ${ }^{[8-18]}$.

Moreover other studies have shown a decrease in microhardness of bleached enamel. A decrease in microhardness might be attributed to the loss of mineral content due to demineralization ${ }^{[19,20,21]}$. Some scientific evidence reports changes in histology and mineral content of bleached enamel. These changes include decreased microhardness and increased porosity and permeability. Changes in bleached enamel may be due to the oxidizing and demineralizing effects of hydrogen peroxide ${ }^{[22-23]}$.

In many studies, artificial saliva was used for storing the specimens after the bleaching cycles, because it is believed that artificial saliva contributed to a slight increase in the microhardness, after demineralization, artificial saliva was used in order to simulate the oral condition ${ }^{[24,25]}$.
On the other hand, a significant increase on mean microhardness after the application of the both remineralizing agent (Clinpro ${ }^{\mathrm{TM}}$ White Varnish and NUPRO Sensodyne with NovaMin). When comparing the effect of both remineralizing agents used, no statistical differences were found.

Clinpro $^{\mathrm{TM}}$ White Varnish contains functionalized tri-calcium phosphate (F-TCP), which has been revealed to have remineralizing result both in vivo and in vitro. F-TCP is formed by the solid state ball milling of beta-tricalcium phosphate and sodium lauryl sulfate. Functionalized tricalcium phosphate prevents calcium from rashly interacting with fluoride and producing calcium fluoride, thus allowing more fluoride and calcium ions to be delivered to the enamel surface. ${ }^{[26]}$ Formation of calcium phosphate or calcium fluoride complexes upon application of FTCP would reduce remineralization by decreasing levels of available calcium and fluoride. To inhibit such effect F-TCP particles are organically coated with sodium lauryl sulphate. Upon contact with saliva the organic component dissolves away leaving the particles active. ${ }^{[27-28]}$

Moreover, functionalized tricalcium phosphateis produced by milling TCP with sodium lauryl sulfate. fTCP stabilizes fluoride in solution and maintains high concentration of calcium, phosphate and fluoride in white spot lesions ${ }^{[9-11]}$. Inclusion of the functionalized TCP ingredient in $\mathrm{NaF}$ formulations resulted in a stronger more acid-resistant mineral relative to fluoride alone in laboratory and clinical evaluations ${ }^{[10]}$.

The result of the current study revealed that a considerable amount of surface microhardness of the enamel is achieved after treatment with Tricalcium phosphate or NovaMin. This is in agreement with a recent study, carried out by Gjorgievska and Nicholson, showed that using the golden standard bioglass 45S5 (NovaMin) incorporated in toothpastes after bleaching could remineralize the enamel surface by increasing the superficial $\mathrm{Ca}$ and 
P contents ${ }^{[18]}$. NovaMin ${ }^{\circledR}$ is a particulate bioactive glass. It is technically known as an inorganic amorphous calcium sodium phosphosilicate (CSPS) material that was designed based on a class of materials known as bioactive glasses. It comprises $45 \% \mathrm{SiO} 2,24.5 \% \mathrm{Na} 2 \mathrm{O}, 24.5 \% \mathrm{CaO}$ and $6 \%$ $\mathrm{P}^{2 \mathrm{O}} 5^{[13]}$.

NUPRO Sensodyne added with NovaMin showed better results, achieving a remineralization up to $2.85 \%$ in calcium, and a significant increase of phosphorus and sodium ions; this corroborates the findings in respect to Vahid Golpayegani et al. ${ }^{[29-30]}$, NovaMin is supposed to release calcium and phosphate ions intra orally to help the selfrepair process of enamel. A silica-rich surface layer forms through poly-condensation of hydrated silica groups on which precipitation of ions happens which crystallizes over time to form a hydroxylcarbonate apatite. Although it is used extensively as a desensitizing agent reports also claim that the chemical reactions that promote apatite formation may enhance the remineralization ${ }^{[30]}$. Following immersion in an aqueous environment, NovaMin releases ions into its surroundings. Sodium ions present in the formulation of calcium sodium phosphor-silicate bioactive glass are replaced with hydrogen ions and thus, the $\mathrm{pH}$ increases. Subsequently, calcium and phosphate ions deposit and form a superficial layer saturated with calcium phosphate on the tooth surface. This ionic reservoir can inhibit the process of demineralization and enhance remineralization by protecting the enamel ${ }^{[12-31-32]}$. Gorgievsk and Nichols, reported that a protective layer of bioactive glass deposits is formed on the enamel surface, and there is an increase in the $\mathrm{Ca}$ and $\mathrm{P}$ content of the enamel surface, returning it to that of undamaged enamel ${ }^{[18]}$. The results of the current study demonstrated that both Clinpro ${ }^{\mathrm{TM}}$ White Varnish (FTCP) and NUPRO Sensodyne with NovaMin are effective in remineralization of bleached enamel surface.

\section{CONCLUSION}

Based on the results of this in vitro study both Tricalcium phosphate and Novamin are effective in remineralization of bleached enamel surface and no significant difference was detected in their efficacy,although the greatest mean value was recorded in Novamin remineralizing agent.

\section{REFERENCES}

1. Ribeiro D.A., Marques M.E.A., Salvadori D.M.F., Study of DNA damage induced by dental bleaching agents in vitro, Braz. Oral Res., 2006, 20, 47-51.

2. Trakiniene G, Daukontiene S, Jurenas V, Svalkauskiene V, Smailiene D, Lopatiene K\& Tomas Trakinis T. The effect of the teeth bleaching with $35 \%$ hydrogen peroxide on the tensile bond strength of metal brackets. Scientific Reports 2017; 7(798):1-6.

3. Furlan SI, Bridi EC, Botelho do Amaral FL, Franca FMG, Turssi CP, Basting RT. Effect of high- or lowconcentration bleaching agents containing calcium and/ or fluoride on enamel microhardness. General Dentistry 2017;65 (3):66-70.

4. Rajesh AG, Ranganath LM, Kumar KS, Rao BS. Surface morphological changes in human enamel following bleaching: An in vitro scanning electron microscopic study. J Contemp Dent Pract. 2012;13(3):405-15.

5. Alaghemand H, Kamangar SSH, Zarenegad N, Tabari N, Abedi H, Khafri S. In-Vitro Effect of Casein Phosphopeptide Amorphous Calcium Phosphate on Enamel Susceptibility to Staining by Tea during Bleaching Treatment. Journal of Dentistry, Tehran University of Medical Sciences. 2015; 12 (8):607-613.

6. Rastelli ANS, Nicolodelli G, Romano RA, D. Milori MBP, Perazzoli ILO, Ferreira EJ, Pedroso ACB, Souza MT, Peitl O, and Zanotto ED. After bleaching enamel remineralization using a bioactive glass-ceramic (BioSilicater). Biomed. Glasses 2016; 2:1-9.

7. Tabatabaei SH, SaraSarhadi, Hosseini Tabatabaei MR, Naebi M. The Effects of remineralizing agents on Microhardness of Bleached Enamel with 40\% Hydrogen Peroxide-An Invitro Study. Sch. J. App. Med. Sci., 2017; 5(4B):1353-1358.

8. Rastelli ANS, Nicolodelli G, Romano RA, Milori DMBP, Perazzoli ILO, Ferreira EJ, Pedroso ACB, Souza MT, Peitl O, and Zanotto ED. After bleaching enamel remineralization using a bioactive glass-ceramic (BioSilicater). Biomed. Glasses 2016; 2:1-9 
9. Sh P, Raghu R, Shetty A, Gautham P, Reddy S, Srinivasan R. Effect of organic versus inorganic fluoride on enamel microhardness: an in vitro study. J Conserv Dent 2013;16:203-207.

10. Asaizumi M, Karlinsey RL, Mackey AC, Kato T, Kuga T. In vitro assessments of white-spot lesions treated with $\mathrm{NaF}$ plus tricalcium phosphate (TCP) toothpastes using microtomography (micro-CT). J Dent Oral Hyg 2013;5:68-76.

11. Mittal R, Relhan N, Tangri T. Remineralizing Agents: A Comprehensive Review. International Journal of Clinical Preventive Dentistry 2017;13(1):1-4.

12. Haghgoo R, Ahmadvand M, Moshaverinia S. Remineralizing Effect of Topical NovaMin and Nanohydroxyapatite on Caries-like Lesions in Primary Teeth. J Contemp Dent Pract 2016;17(8):645-649.

13. Kumar A, Singh S, Thumar G, Mengji A. Bioactive Glass Nanoparticles (NovaMin $\AA$ ) for Applications in Dentistry. IOSR Journal of Dental and Medical 2015;14 (8):30-35

14. Robin A \& Meirelis JP (2007) Influence of fluoride concentration and $\mathrm{pH}$ on corrosion behavior of titanium in artificial saliva Journal of Applied Electrochemistry 37(4) 511-517.

15. Borges AB, Yui KC, D'Avila TC, Takahashi CL, Torres $\mathrm{CR}$, Borges $\mathrm{AL}$. Influence of remineralizing gels on bleached enamel microhardness in different time intervals. Oper Dent 2010;35:180-186.

16. Ghanbarzadeh M, Ahrari F, Akbari M, Hamzei H. Microhardness of demineralized enamel following home bleaching and laser-assisted in office bleaching. J Clin Exp Dent. 2015;7(3):405-409.

17. Janavathi, Rajnikanth, Jyothi L, Naveen Kumar and Tirumal Rao T. The Effect of McInnes Solution on Enamel and the Effect of GC Tooth Mousse on Bleached Enamel: An In Vitro Study. Indian Journal of Mednodent and Allied Sciences 2014; 2 (1):21-28.

18. Gjorgievska E., Nicholson J.W., Prevention of enamel demineralization after tooth bleaching by bioactive glass incorporated into toothpaste, Aust. Dent. J., 2011, 56(2), 193-200.

19. Cunha AG, de Vasconcelos AA, Borges BC, Vitoriano Jde O, Alves-Junior C, Machado CT, dos Santos AJ. Efficacy of in "office bleaching techniques combined with the application of a casein phosphopeptide" amorphous calcium phosphate paste at different moments and its influence on enamel surface properties. Microsc Res Tech 2012;75:1019-1025.
20. Maryam Khoroushi1,Farinaz Shirban2, Sara Kaveh3, Samaneh Doustfateme4* Khoroushi M, Shirban F, Kaveh $S$, Doustfateme S.Effect of three nanobiomaterials on microhardness of bleached enamel. Restor Dent Endod 2016; 41(3):196-201

21. Alaghemand H, Kamangar SSH, Zarenegad N, Tabari $\mathrm{N}$, Abedi H, Khafri S. In-Vitro Effect of Casein Phosphopeptide Amorphous Calcium Phosphate on Enamel Susceptibility to Staining by Tea during Bleaching Treatment. Journal of Dentistry, Tehran University of Medical Sciences 2015;12(8):607-613.

22. Petta TM, Gomes YS BL, Esteves RA, Faial KCF, Couto RSA and Silva CM. Chemical Composition and Microhardness of Human Enamel Treated with Fluoridated Whitening Agents. A Study in Situ. Dentistry Journal 2017;11:34-40

23. COCESKA E, GJORGIEVSKA E, NICHOLA J, COLEMAN, GABRIC D SLIPPER IJ, STEVANOVIC M \& NICHOLSON JW. Enamel alteration following tooth bleaching and remineralization. Journal ofMicroscopy, 2015; 00, (0):1-13

24. Iijima Y, Cai F, Shen P, Walker G, Reynolds C, Reynolds EC. Acid resistance of enamel subsurface lesions remineralized by a sugar-free chewing gum containing casein phosphopeptide-amorphous calcium phosphate. Caries Res. (2004). 38: 551-6.

25. Chow LC,Takagi S, Carey CM,SieckBA.Remineralization effects of a two- solution fluoride mouthrinse: An in situ study. J Dent Res. (2000). 79: 991-995.

26. Vanichvatana S, Auychai P. Efficacy of two calcium phosphate pastes on the remineralization of artificial caries: a randomized controlled double-blind in situ study. Inter J Oral Sci. 2013; 5: 224-228.

27. Karlinsey RL, Mackey AC. Solid-state preparation and dental application of an organically modified calcium phosphate. J Mater Sci 2009; 44: 346-349.

28. Nagi SM, El Hoshy AZ, Elenin KA. Effect of two remeniralizing agents on the microhardness and ultramorphology of deminaralized enamel. Medicine Sciences and Healthcare Journal 2015;10 (12):2-12.

29. Vahid Golpayegani M, Sohrabi A, Biria M, Ansari G. Remineralization Effect of Topical NovaMin Versus Sodium Fluoride $(1.1 \%)$ on Caries-Like Lesions in Permanent Teeth Journal of Dentistry, Tehran University of Medical Sciences 2012; 9( 1):68-75. 
30. Carrillo EL, Chimal CD, González SL, Luckie RAM, Olea-Mejía OF, Kubodera-Ito T, Medina-Solís CE. Remineralization effect of low-level laser and amorphous sodium-calcium-phosphosilicate paste in teeth with fixed orthodontic appliances. Tanta Dental Journal 2016; 13:55-62.
31. RehderNeto FC, Maeda FA, Turssi CP, Serra MC. Potential agents to control enamel caries-like lesions. J Dent 2009; 37(10):786-790

32. Rai R, Verma S, Bhardwaj A. NovaMin ${ }^{\circledR}$ : A promising anti-hypersensitivity agent. SPER Journal of Pharmacy Research 2016; (1): 1-3. 